LANL e-print gr-qc/9802041

Int. J. Mod. Phys. D 8 (1999) 549-555

\title{
Barotropic thin shells with linear EOS as models of stars and circumstellar shells in general relativity
}

\author{
Konstantin G. Zloshchastiev \\ Box 2837, Dnepropetrovsk 320128, Ukraine; e-mail: zlosh@email.com
}

\begin{abstract}
The spherically symmetric thin shells of the barotropic fluids with the linear equation of state are considered within the frameworks of general relativity. We study several aspects of the shells as completely relativistic models of stars, first of all the neutron stars and white dwarfs, and circumstellar shells. The exact equations of motion of the shells are obtained. Also we calculate the parameters of the equilibrium configurations, including the radii of static shells. Finally, we study the stability of the equilibrium shells against radial perturbations.
\end{abstract}

PACS number(s): 04.40.Nr, 11.27.+d, 12.38.Mh, 97.10.Fy

Keyword(s): general relativity, thin shell, star, circumstellar shell 
In present paper we consider some infinitely thin (singular) shells as models of the physical entities, which thickness is negligible in comparison with a circumference radius (e.g., the circumstellar shells or surfaces of phase transitions). Besides, one may study the shells as the simplest (nevertheless useful and instructive) models of "thick" objects, e.g., stars. Geometrically the shell is described by a three-dimensional closed hypersurface, embedded in the four-dimensional spacetime and dividing it into the two domains, the external $\left(\Sigma^{+}\right)$ and internal $\left(\Sigma^{-}\right)$spacetime. Since the classical works [1], 2] the theory of surface layers has been widely considered in the literature (see ref. [3] for details) including modern applications and developments in fundamental, quantum and phenomenological directions, see ref. [四 and references therein. We only point out some questions of principle now. One of them is the essential difference between the description of the boundary surface (for instance, surface of star) and shell. The boundary surface is the discontinuity of the first kind (mass density has a finite jump across the surface) and is described by the Lichnerowicz-Darmois junction conditions (the first and second quadratic forms are continuous on the surface). The singular thin shell is the discontinuity of the second kind (the density has the delta-like singularity on the shell) and is described by the Lichnerowicz-Darmois-Israel junction conditions: the first quadratic form (the metric) is continuous, the second one (the extrinsic curvature) has a finite jump.

This circumstance says we must take care at physical interpretation (and application) of the shells. For instance, the surface of a star should be described by a boundary surface not a singular thin shell. Latter can be used as a model: (a) of the star as a whole system [5], we replace a thick layer of matter (which has an infinite number of degrees of freedom) by the singular shell which has a finite number of collective degrees of freedom (spherically symmetric thin shells have only radial degree of freedom), (b) of the stellar internal structure, thereby a star can be considered as a set of an infinite number of embedded shells, (c) of the circumstellar shells as a set of a finite number of shells. The aim of this paper is to study all appropriate cases for the simplest class of equations of state (EOS).

So, one considers a thin shell with the surface stress-energy tensor of a perfect fluid in the general case

$$
S_{a b}=\sigma u_{a} u_{b}+p\left(u_{a} u_{b}+{ }^{(3)} g_{a b}\right),
$$

where $\sigma$ and $p$ are the surface energy density and pressure respectively, $u^{a}$ is the timelike unit tangent vector, ${ }^{(3)} g_{a b}$ is the 3-metric of a shell's surface. We suppose metrics of the spacetimes outside $\Sigma^{+}$and inside $\Sigma^{-}$of a spherically symmetric shell to be of the Schwarzschild form, i.e.,

$$
\mathrm{d} s_{ \pm}^{2}=-\left[1-\frac{2 M_{ \pm}}{r}\right] \mathrm{d} t_{ \pm}^{2}+\left[1-\frac{2 M_{ \pm}}{r}\right]^{-1} \mathrm{~d} r^{2}+r^{2} \mathrm{~d} \Omega^{2}
$$

where $\mathrm{d} \Omega^{2}$ is the metric of the unit 2-sphere. It corresponds to the neutral shell surrounding a neutral body of mass $M_{-}$, and $M_{+}$appears to be the external observable total mass (energy) of the whole system. We assume both masses to be non-negative (though a theory of quantum gravity could give rise to spacetimes of negative mass, see ref. [4, 6]). It is possible to show 
that if one uses the proper time $\tau$ then the 3 -metric of a shell is

$$
{ }^{(3)} \mathrm{d} s^{2}=-\mathrm{d} \tau^{2}+R^{2} \mathrm{~d} \Omega^{2}
$$

where $R=R(\tau)$ is a proper radius of a shell. Also the energy conservation law for matter on a shell (which is an integrability condition for the Einstein equations on a shell) can be written as

$$
\mathrm{d}\left(\sigma^{(3)} g\right)+p \mathrm{~d}\left({ }^{(3)} g\right)=0
$$

where ${ }^{(3)} g=\sqrt{-\operatorname{det}\left({ }^{(3)} g_{a b}\right)}=R^{2} \sin \theta$. In this equation, the first term corresponds to a change in the shell's internal energy, the second term corresponds to the work done by the shell's internal forces.

Imposing junction conditions across a shell, the Einstein equations yield the equation of shell's motion in the form

$$
\epsilon_{+} \sqrt{1+\dot{R}^{2}-\frac{2 M_{+}}{R}}-\epsilon_{-} \sqrt{1+\dot{R}^{2}-\frac{2 M_{-}}{R}}=-\frac{m}{R},
$$

where

$$
m=4 \pi \sigma R^{2}
$$

is interpreted as an effective rest mass, $\dot{R}=\mathrm{d} R / \mathrm{d} \tau$ is the proper velocity of a shell, $\epsilon_{ \pm}=$ $\operatorname{sign}\left(\sqrt{1+\dot{R}^{2}-2 M_{ \pm} / R}\right)$. Equations (4) - (6) together with an equation of state and choice of the signs $\epsilon_{ \pm}$, completely determine the motion of the fluid Schwarzschild shell in general relativity. Therefore, we must resolve the next two problems, viz., the choice of $\epsilon_{ \pm}$in eq. (5), and the choice of EOS.

(i) The choice of signs $\epsilon_{ \pm}$. It is well-known, that $\epsilon=+1$ if $R$ increases in the outward normal direction to the shell ( e.g., it takes place in a flat spacetime), and $\epsilon=-1$ if $R$ decreases (semiclosed world). Thus, only under the additional condition $\epsilon_{+}=\epsilon_{-}=1$ we have an ordinary shell [7, 8]. In the paper we model ordinary stars not the wormhole-stars, therefore, in (5) we suppose $\epsilon_{+}=\epsilon_{-}=1$.

(ii) The choice of the equation of state. We confine our discussion to models calculated with ultra-high density barotropic state equations of the linear form

$$
{ }^{(3)} p=(\gamma-1)^{(3)} \varepsilon
$$

For physical purposes the cores with $1<\gamma \leq 2$, e.g., $\gamma=2,4 / 3,6 / 5,14 / 13$ were considered [9] as models of neutron stars. They showed a maximum mass of approximately $0.7 M_{\odot}$ corresponding to a radius around four Schwarzschild radii. For two-dimensional matter eq. (1) implies

$$
p=\eta \sigma
$$

where the reduction of dimensionality of the stress-energy tensor should be taken into account, i.e., $2 \eta=3(\gamma-1)$. This equation includes the most studied cases: the dust shell $p=0$ 
[2, 10], radiation fluid shell $\sigma-2 p=0$ [11], and bubble $\sigma+p=0$ [7, 12]. If $\eta>0$ it can be interpreted as a square component of the vector of a speed of sound in the shell. Then for a spatially two-dimensional homogeneous fluid the square speed of sound is $2 \eta$. From the physical viewpoint some $\eta$ appear to be inadmissible. For instance, if the fluid on a shell is required to satisfy the dominant energy condition, $\sigma \geq|p|$, one obtains the constraint

$$
|\eta| \leq 1
$$

If a fluid is required to satisfy the causality condition, we get the constraint

$$
\eta \leq 1 / 2
$$

where one took into account spatial two-dimensionality of the fluid. Nevertheless, the aim of this paper is to study the general case of arbitrary $\eta[13]$. So, solving the differential equation (4) with respect to $\sigma$, we obtain

$$
\sigma=\frac{C}{4 \pi} R^{-2(\eta+1)}
$$

where $C$ is the integration constant determined by the specific shell matter. The value of $C$ is closely related to the value of surface mass density (or pressure) at fixed $R$. We consider ordinary shells not wormholes, therefore, we require $\sigma>0$, hence $C>0$. Also it should be noted that from (6) at positive densities $\sigma$ it follows that $M_{+}>M_{-}$for any $R$ and $\dot{R}$. Otherwise, matching of the spacetimes (2) is impossible. Thus, we have all necessary equations (5), (6) and (11) to study the shells as models of stars.

First of all we study the static shells. Taking into account (8), the equilibrium conditions $\dot{R}=\ddot{R}=0$ [14 for our case read

$$
\begin{gathered}
k_{-}-k_{+}=4 \pi \sigma_{0} r_{0}, \\
\frac{M_{+}}{k_{+}}-\frac{M_{-}}{k_{-}}=4 \pi(2 \eta+1) \sigma_{0} r_{0}^{2},
\end{gathered}
$$

where $k_{ \pm}=\sqrt{1-2 M_{ \pm} / r_{0}}\left(k_{+}<k_{-}\right), r_{0}$ and $\sigma_{0}$ are the shell radius $R$ and density at equilibrium respectively, $\sigma_{0}$ is given by (11) at $R=r_{0}$. Taking into account (12), the equation $(13)$ can be rewritten in the nice form

$$
\frac{1}{k_{+} k_{-}}=4 \eta+1
$$

We can use the system (12), (14) instead (12), (13). The equation (14) is very helpful because it already gives us both the radius $r_{0}$ and constraint for $\eta$ independently of the arbitrary constant $C$.

Proposition. Let we have the neutral ordinary shell of the matter with the EOS (8), $k_{ \pm}$ are real and positive, $M_{+} \neq M_{-}$. Then the shell can have static states only at

$$
\eta>0 .
$$


Proof: From eq. (14) it follows that $4 \eta+1>0$, and

$$
1-\frac{(4 \eta+1)^{-2}}{1-2 M_{-} / r_{0}}=\frac{2 M_{+}}{r_{0}}>0,
$$

or

$$
(4 \eta+1)^{-2}<1
$$

These conditions give the desired inequality $\eta>0$, Q.E.D.

Hence it follows that neither the dust shells $(\eta=0)$, nor bubbles $(\eta=-1)$ can have static states with mass $M_{+}$. In other words, they should not be considered as static sources of the Schwarzschild metric (2). By means of this proposition we can prove the next theorem.

Theorem. Let the conditions of the previous proposition are valid. Then the radius of static shells is given by the expression

$$
r_{0}=\frac{1}{\varrho}\left[M_{+}+M_{-}+\sqrt{\Delta M^{2}+4 M_{+} M_{-}(1-\varrho)}\right],
$$

where $\varrho=1-(4 \eta+1)^{-2}, \Delta M=M_{+}-M_{-}$.

Proof: Perform the conformal transformation $\xi=r_{0} / 2 M_{+}, \alpha=M_{-} / M_{+}$. The case $\alpha=0$ corresponds to the hollow shell $\left(M_{-}=0\right)$, but $\alpha=1$ describes the limit case $M_{ \pm} \rightarrow \infty$, $\Delta M \rightarrow 0$ (infinitely light shells), not the case $M_{+} \equiv M_{-}$which for ordinary shells is forbidden as trivial (5). Therefore $0 \leq \alpha \leq 1$. Then, squaring eq. (14), we obtain the square equation with respect to $\xi$

$$
\varrho \xi^{2}-(1+\alpha) \xi+\alpha=0
$$

which has the two roots

$$
2 \varrho \xi_{ \pm}=1+\alpha \pm \sqrt{(1+\alpha)^{2}-4 \alpha \varrho}
$$

one of them is superfluous, as can be directly verified. The preceding proposition yields

$$
0<\varrho<1, \sqrt{(1+\alpha)^{2}-4 \alpha \varrho}<1+\alpha .
$$

Further, taking into account eq. (16), the condition $\xi>1$ can be written as

$$
\pm \sqrt{(1+\alpha)^{2}-4 \alpha \varrho}>2 \varrho-1-\alpha .
$$

If we define the auxiliary function

$$
z_{ \pm}(\varrho)=1+\alpha-2 \varrho \pm \sqrt{(1+\alpha)^{2}-4 \alpha \varrho}
$$

then we must require $z_{ \pm}(\varrho)>0$. It can easily be seen that

$$
z_{+}(\varrho \neq 1)>0, z_{+}(1)=0, z_{-}(\varrho \neq 0)<0, z_{-}(0)=0,
$$

therefore, only the root $\xi_{+}$is admissible, Q.E.D. 
The radius $r_{0}$ given by eq. (15) is illustrated in Fig. 1 for the most physically admissible range, 0 (dust) $<\eta \leq 1 / 2$ (ultrarelativistic fluid). For clarity only the two limit cases, $\alpha=0$ and $\alpha=1$, are represented, the curves for other $0<\alpha<1$ lie between them. The parameter $1-\alpha=\Delta M / M_{+}$determines the proper total mass of the shells. As it was mentioned above, we can model both the whole star and stellar internal structure. In this connection the model with $\alpha=0$ (the hollow shell) appears to be a model of the star as a whole system, whereas the shells at $\alpha \neq 0$ can be models both inhomogeneous stellar structure (the layers with smaller $\eta$ lie above those with greater $\eta$ ) and the circumstellar shells.

Another important aspect we must consider is the mechanical stability of the shells. Here we apply the method developed in ref. [14. Redefining the constant $C$ in (11), we suppose

$$
\frac{\sigma}{\sigma_{0}}=\frac{1}{4 \pi} z^{-2(\eta+1)}
$$

where $z=R / r_{0}$, the constants $\sigma_{0}$ and $r_{0}$ should be calibrated by means of the system (12), (14). It is well-known that the shell's equation of motion can be rewritten in a more convenient form. Squaring (5) twice, one obtains

$$
\dot{R}^{2}+V(R)=0
$$

where the potential $V(R)$ is

$$
V(R)=1-\frac{2 M_{+}}{R}-\left[\frac{a}{R}\left(\frac{R}{r_{0}}\right)^{2 \eta+1}-\frac{\Delta k}{2}\left(\frac{r_{0}}{R}\right)^{2 \eta+1}\right]^{2},
$$

where $a=\Delta M / \Delta k, \Delta k=k_{+}-k_{-}$. Calibrating these expressions, we obtain

$$
r_{0}^{2} \dot{z}^{2}+V(z)=0
$$

where

$$
V(z)=1+\frac{k_{+}-1}{z}-\left[\frac{\varrho\left(k_{+}^{2}+1\right) z^{4 \eta+1}+\varrho k_{+}^{2}-1}{2 \varrho k_{+} z^{2 \eta+1}}\right]^{2} .
$$

The potential satisfies $V(z=1)=\mathrm{d} V /\left.\mathrm{d} z\right|_{z=1}=0$, as can be directly verified. Therefore, the stability of the equilibrium is equivalent to $V(z)$ having a minimum at $z=1$. By differentiation one obtains

$$
\left.\frac{\mathrm{d}^{2} V}{\mathrm{~d} z^{2}}\right|_{z=1}=-\frac{k_{+}^{4}(4 \eta+1)^{2}-2 k_{+}^{2}+1}{2 k_{+}^{2}} .
$$

From eq. (21) and from the proposition proved above it can readily be seen that $\mathrm{d}^{2} V /\left.\mathrm{d} z^{2}\right|_{z=1}<$ 0 . Therefore, the equilibrium of the neutral shells with the linear EOS (unlike those with the linearized EOS studied by Brady, Louko and Poisson [14]) is unstable against radial perturbations. In application to models of neutron stars it means that the EOS (8) can not 
purely describe them. Apparently only the average layers of the star core can be "made" from the matter (8). The central part of the core (the so called "core of the core") and core's crust must be consisting of other kind substance to prevent from collapse into black holes and explosion respectively. For instance, Witten [15] argued that the strange matter can be formed at the quark-hadron phase transition in central part of neutron stars. As for the crust that it can be even crystallic [16].

Thus, in the present paper some aspects of dynamics of the spherically symmetric shells with the linear EOS were considered. Finally it should be noted that the studied shells may also describe, besides static (or having adiabatically slow radial motion) circumstellar shells and stars, the transient astrophysical phenomena, e.g., the blast waves at supernova explosion. Thereby the obtained models of these phenomena will be completely relativistic that is essential at high velocities and powerful fields when the spacetime can not be supposed flat. 


\section{References}

[1] G. Dautcourt, Math. Nachr. 27 (1964) 277.

[2] W. Israel, Nuovo Cimento 44B (1966) 1.

[3] C. W. Misner, K. S. Thorne and J. A. Wheeler, Gravitation (Freeman, San Francisco, 1973).

[4] K. G. Zloshchastiev, Gen. Relativ. Gravit. 31 (1999) 571; Int. J. Mod. Phys. D 8 (1999) 165; ibid. 8 (1999) 363; Acta Phys. Polon. B 30 (1999) 897; Class. Quantum Grav. 16 (1999) 1737.

[5] D. Núñez, Astrophys. J. 482 (1997) 963.

[6] R. Mann, Class. Quantum Grav. 14 (1997) 2927.

[7] P. Laguna-Castillo and R. A. Matzner, Phys. Rev. D 34 (1986) 2913; V. A. Berezin, V. A. Kuzmin and I. I. Tkachev, Phys. Rev. D 36 (1987) 2919.

[8] V. A. Berezin, V. A. Kuzmin and I. I. Tkachev, Phys. Lett. B 120 (1983) 91; V. A. Berezin, N. G. Kozimirov, V. A. Kuzmin and I. I. Tkachev, Phys. Lett. B 212 (1988) 415; D. S. Goldwirth and J. Katz, Class. Quantum Grav. 12 (1995) 769.

[9] C. W. Misner and H. S. Zapolsky, Phys. Rev. Lett. 12 (1964) 635.

[10] P. Hájíček and J. Bičák, Phys. Rev. D 56 (1997) 4706; J. L. Friedman, J. Louko and S. N. Winters-Hilt, ibid. 56 (1997) 7674; K. G. Zloshchastiev, ibid. 57 (1998) 4812.

[11] M. Visser, Nucl. Phys. B 328 (1989) 203.

[12] S. Coleman and F. De Luccia, Phys. Rev. D 21 (1980) 3305.

[13] K. G. Zloshchastiev, Mod. Phys. Lett. A 13 (1998) 1419.

[14] J. Frauendiener, C. Hoenselaers and W. Konrad, Class. Quantum Grav. 7 (1990) 585; P. R. Brady, J. Louko and E. Poisson, Phys. Rev. D 44 (1991) 1891.

[15] E. Witten, Phys. Rev. D 30 (1984) 272.

[16] Ya. B. Zel'dovich and I. N. Novikov, Theory of gravity and evolution of stars (Nauka, Moskow, 1971). 
Figure 1: Plot of the static shell radius (in units of the Schwarzschild radius) vs $\eta$. The dashed curve describes hollow shells $(\alpha=0)$, the solid curve does the asympotical case $\alpha=1$ (infinitely light shells). The shaded region corresponds to black holes. 
This figure "fig.jpg" is available in "jpg" format from: http://arxiv.org/ps/gr-qc/9802041v4 